Preprints of the $7^{\text {th }}$ IFAC Advances in Control Education (ACE), (Eds. Dormido, Fernández, Morilla, Pastor), CD-Rom M-26008-2006, Madrid-Spain, 6p, 2006

\title{
SELF-ASSESSMENT OF RESPIRATORY MECHANICS AS EDUCATIONAL TOOL IN SYSTEM IDENTIFICATION COURSE
}

\author{
Clara Ionescu and Robin De Keyser \\ Ghent University, EeSA Department of Electrical energy, Systems and Automation, \\ Technologiepark 913, B9052 GENT, Belgium \\ Email address: clara@autoctrl.UGent.be,rdk@autoctrl.UGent.be
}

\begin{abstract}
In this contribution, a project task is presented for master students that follow the course on advanced control techniques. The aim of the project is to make students familiar with the non-parametric identification techniques - via spectral analysis. This method is usually very abstract, theoretical and un-appealing to the students. Therefore, an interesting application is proposed: identification of their own respiratory system mechanics. Issues on data acquisition, sampling frequency, resolution, accuracy and noise trade-offs are analyzed. The students are allowed to work in team - promoting thus brainstorm sessions - but each of them has to submit a report with his/her own respiratory impedance analysis. Copyright (C) 2006 IFAC
\end{abstract}

Keywords: Accuracy, Biomedical systems, Education, Fast Fourier transform, Nonparametric identification, Signal processing, Spectral analysis, Resolution

\section{INTRODUCTION}

Quality teaching of identification and control techniques involves good theory fundamentals applied on practical examples. At the end of their studies, the students must achieve the knowledge how to apply the theory from the course in real-life experiments. Therefore, a good balance between theory and practice has a crucial role in the education (Wellstead, 1990).

Some of the non-parametric identification procedures, such as the frequency response analysis, are rather abstract (Ljung, 1999). These procedures contain a significant amount of mathematical considerations, making them less attractive to control engineering students - by definition a practice oriented study field. In order to reduce the abstractness of this topic and make it more attractive to the students, a project work is proposed in which the system to be identified is the student's own respiratory function. Simultaneously, dealing with real-life aspects (such as sampling frequency, resolution, disturbances, etc) is a good training for their future engineering career.

The aim of the project work is to estimate the frequency response characteristic of the respiratory system by use of non-parametric identification methods. Spectral analysis is applied to obtain an estimate of the respiratory impedance of a subject (by this definition, each student is a subject). A non invasive lung function test (Forced Oscillations Technique - FOT) and an experimental set-up, are used to measure the respiratory pressure and the respiratory flow involved in the respiratory mechanics of the subject.

\section{PROJECT TASK}

The task of the project consists of estimating the respiratory impedance of each student by use of nonparametric identification techniques. The students use a non-invasive lung function test - forced oscillations technique FOT - to excite the respiratory system. The input - pressure oscillations - and the 
output - trans-respiratory air pressure and air flow are available as raw data vector for further processing. Via signal processing techniques spectral analysis - the frequency response of the respiratory system is obtained by means of Bode (magnitude and phase) and Nyquist plots (real and imaginary parts of the respiratory impedance).

FOT is applied to the patient (student) by use of a commercial apparatus available in our laboratory modified for the purpose of this experiment. This device is able to provide the raw measured signals (input-output), making them available for signal processing. The students are then invited to apply the non-parametric identification methods (via correlation analysis and via averaged modified periodograms) and obtain the respiratory impedance of their lungs.

Since there is no universal model-solution due to the fact that each subject has its personal respiratory impedance, the validation is possible only by use of the commercial FOT device. The device provides an estimation of respiratory impedance for each patient that performs the FOT test - which can then be used as a validation set.

\section{THE RESPIRATORY SYSTEM}

In this project work, each student will identify his/her own respiratory system by means of frequency-response analysis. The frequency response of the respiratory system gives insight upon the mechanical properties of the lungs, thus an analysis of the lung function (Navajas and Farré, 1999).

The mechanical properties of the respiratory system may be obtained by applying the forced oscillation technique (FOT), and measuring the resulting airflow (Q) and trans-respiratory pressure (P). As the application of this non-invasive technique does not require the patient's cooperation (except for breathing!), it is suitable for routine evaluation of respiratory function (Oostveen et al., 2003). However, it should be pointed out that with FOT respiratory mechanics is assessed at a frequencyrange $(4-48 \mathrm{~Hz})$, which is higher than the spontaneous breathing frequency (around $0.2 \mathrm{~Hz}$ for a normal pulmonary function).

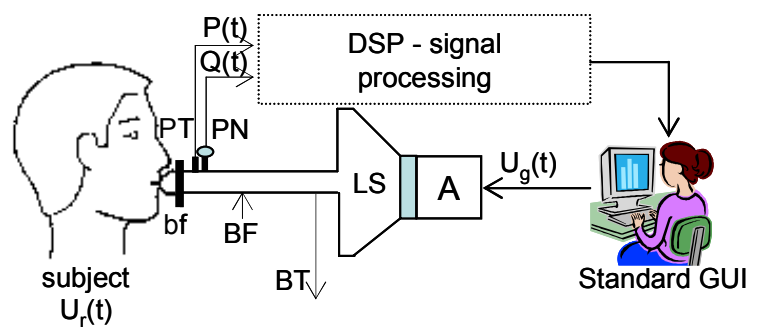

Fig. 1. Standard FOT set-up. The following notations apply: DSP - digital signal processing board; LS - loudspeaker; BT - bias-tube; BF - bias-flow; PN - pneumotachograph; bf - biological filter; A amplifier; PT - pressure transducer; $\mathrm{Q}$ - flow and $\mathrm{P}$ - pressure, $\mathrm{U}_{\mathrm{g}}$ - the generated input; $\mathrm{U}_{\mathrm{r}}-$ the breathing signal.
The conventional / commercially available FOT setup is based on superimposing a low-amplitude pressure oscillation at the mouth while the patient is breathing spontaneously as illustrated in Figure 1.

The oscillation pressure is generated by a loudspeaker (LS) connected to a chamber, driven by a power amplifier fed with the oscillating signal generated by a computer. The movement of the loudspeaker cone generates a pressure oscillation inside the chamber, which is applied to the patient's respiratory system by means of a tube connecting the loudspeaker chamber and the mouthpiece (bf). As the patient breathes spontaneously through a bias tube (BT) - ideally presenting low impedance to the breathing frequency and high impedance to the forced oscillation frequency - a constant bias flow (BF) avoids re-breathing. It is advisory that during the measurements, the patient should wear a noseclip and keep the cheeks firmly supported.

Pressure and flow are measured at the mouthpiece by means of 1) a pressure transducer (PT), and 2) a pneumotachograph (PN) plus a differential pressure transducer (PT), respectively. The signals are analogically low-pass filtered, sampled, stored and processed in a digital signal processing board (DSP).

Both the oscillatory pressure $\mathrm{P}(\mathrm{t})$ and air-flow $\mathrm{Q}(\mathrm{t})$ are periodic with the same period $\mathrm{T}$ (of breathing). The modulus of oscillatory impedance $\left(\left|Z_{\mathrm{r}}\right|\right)$ is defined as the quotient between the amplitude of oscillatory pressure and the amplitude of oscillatory flow. The higher the $\left|Z_{r}\right|$, the greater the pressure amplitude required to induce a given flow. Thus, $\left|Z_{\mathrm{r}}\right|$ is a measure of the total mechanical load of the respiratory system at the oscillation frequency. The phase of respiratory impedance $\left(\Phi_{\mathrm{r}}\right)$ is defined as the phase lag between $\mathrm{P}(\mathrm{t})$ and $\mathrm{Q}(\mathrm{t})$ and it is computed as the ratio between the time lag and the oscillation period $\left(\Phi_{\mathrm{r}}=360^{\circ} \times \Delta \mathrm{t} / \mathrm{T}\right)$. The frequency where $\Phi_{\mathrm{r}}$ $=0$ is called the «resonance frequency» and it depends on the balance between the different kind of mechanical properties (resistive, elastic, inertial) determining thus the mechanics of the respiratory system (Van Noord, 1990).

\section{MEASUREMENT AND INSTRUMENTATION}

The lung function test performed by the standard FOT set-up apparatus is provided with a GUI (graphical user-interface) on the desktop computer software (figure 1). When activating the measurement procedure, a driving signal (multisine from $4-48 \mathrm{~Hz}$ ) is send to the loudspeaker and further onto the mouthpiece and to the subject. The data measurement with the GUI lasts for 8 seconds and afterwards provides the frequency response characteristic of the subject by means of real and imaginary parts (see next section for details). The numerical values of the Real and Imaginary Part vectors in the given frequency points are provided.

In order to perform their own estimation by use of spectral analysis, the students must measure the test 
in $\mathrm{MatLab}^{\circledR}$ environment. This is done in parallel with the standard GUI measurement, by use of a data acquisition board (PCMCIA card, 12 bit, NI6024E series) in a laptop available to the students. An external connection of the measured signals from the FOT apparatus is provided for this purpose and the data is recorded (see figure 2) as vectors in MatLab ${ }^{\circledR}$ environment, at a sampling frequency of $500 \mathrm{~Hz}$ $(=2 \mathrm{~ms})$. The input signal is forced oscillation pressure of maximum $1 \mathrm{cmH}_{2} \mathrm{O}$ amplitude.
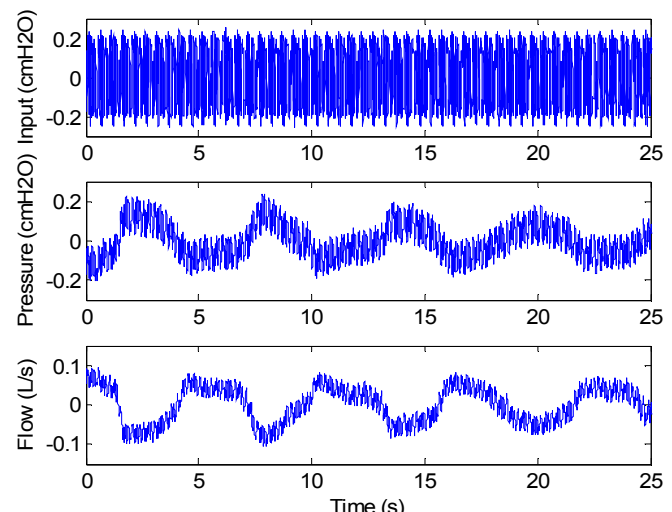

Fig. 2. Measured input and output time signals.

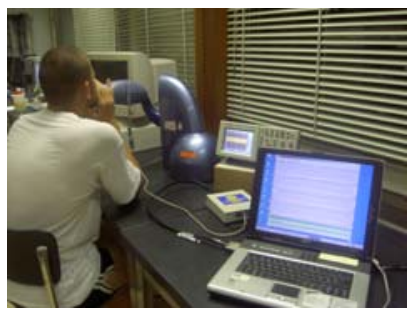

Fig. 3. Real-life measurement setup.

The parallel measurement is less limited in terms of measured number of samples than the FOT-DSP board. In this case, 12500 samples at 0.002 s resulting in 25 seconds time interval is considered optimal, providing good spectral resolution and accuracy. The real-life setup is depicted in figure 3. The students are working in groups of 3-4 in order to perform the experiments. Each student performs three experiments and an average is taken into account for the respiratory systems analysis, avoiding thus intrapatient variability issues (Oostveen et al., 2003). At the end of the measurement and analysis, each student must submit a report with his/her own respiratory function characteristics.

\section{SYSTEM ANALYSIS}

The global experimental set-up can be modelled by the electrical analogy of Fig. 4. Using the basic laws for analysing electrical networks, the following relationships can be derived:

$$
\begin{gathered}
P=\frac{\left(Z_{m}-Z_{3}\right) Z_{r}}{\left(Z_{m}+Z_{r}\right) Z_{1}} U_{g}+\frac{Z_{m}}{Z_{m}+Z_{r}} U_{r} \\
Q=\frac{Z_{m}-Z_{3}}{\left(Z_{m}+Z_{r}\right) Z_{1}} U_{g}-\frac{1}{Z_{m}+Z_{r}} U_{r}
\end{gathered}
$$

with

$$
Z_{m}=Z_{3}+\frac{Z_{1} Z_{2}}{Z_{1}+Z_{2}}
$$

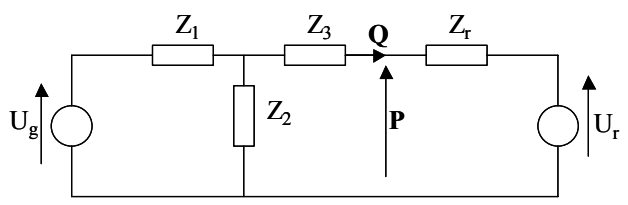

Fig. 4. Electrical scheme analogy. $U_{g}=$ generator test signal - driving signal (measured); $U_{\mathrm{r}}=$ effect of spontaneous breathing (respiratory system / unknown); $Z_{\mathrm{r}}=$ impedance of interest (to be estimated): the impedance of the total respiratory system (including the airways, lung tissues and chest wall); $Z_{1}=$ impedance (unknown) describing the transformation of driving voltage $\left(\mathrm{U}_{\mathrm{g}}\right)$ to chamber pressure; $Z_{2}=$ impedance (unknown) of both bias tubes and loud-speaker chamber; $Z_{3}=$ impedance (unknown) of tube segment between bias tube and mouth piece (effect of pneumotachograph essentially); $\mathrm{P}=$ (measured) pressure; $\mathrm{Q}=$ (measured) flow.

This is a system

$$
\left[\begin{array}{l}
P(s) \\
Q(s)
\end{array}\right]=\underline{H}(s)\left[\begin{array}{c}
U_{g}(s) \\
U_{r}(s)
\end{array}\right]
$$

with 2 inputs $U_{g}$ and $U_{r}, 2$ outputs $P$ and $Q$ and transfer matrix:

$$
\underline{H}=\left[\begin{array}{ll}
\frac{\left(Z_{m}-Z_{3}\right) Z_{r}}{\left(Z_{m}+Z_{r}\right) Z_{1}} & \frac{Z_{m}}{Z_{m}+Z_{r}} \\
\frac{Z_{m}-Z_{3}}{\left(Z_{m}+Z_{r}\right) Z_{1}} & \frac{-1}{Z_{m}+Z_{r}}
\end{array}\right]
$$

(all impedances $Z_{*}$ being also a function of ' $s$ '; the symbol 's' denotes Laplace-operator). Define now the vectors:

$$
\underline{S}_{Y U}=\left[\begin{array}{l}
S_{P U_{g}} \\
S_{Q U_{g}}
\end{array}\right] \text { and } \quad \underline{S}_{U U}=\left[\begin{array}{c}
S_{U_{g} U_{g}} \\
S_{U_{r} U_{g}}
\end{array}\right]
$$

containing cross-power-spectra $\mathrm{S}_{\mathrm{yu}}(\mathrm{j} \omega)$ between 2 signals $y(t)$ and $u(t)$ and auto-power-spectra $S_{u u}(\omega)$ of a signal $u(t)$. From identification and signalprocessing theory it then follows that:

$$
\underline{S}_{Y U}(j \omega)=\underline{H}(j \omega) \underline{S}_{U U}(j \omega)
$$

In case of 'absence of breathing' $\left(\mathrm{U}_{\mathrm{r}}=0\right)$ the expression (7) reduces to:

$$
\left[\begin{array}{l}
S_{P U_{g}} \\
S_{Q U_{g}}
\end{array}\right]=\left[\begin{array}{c}
\frac{\left(Z_{m}-Z_{3}\right) Z_{r}}{\left(Z_{m}+Z_{r}\right) Z_{1}} \\
\frac{\left(Z_{m}-Z_{3}\right)}{\left(Z_{m}+Z_{r}\right) Z_{1}}
\end{array}\right] \cdot S_{U_{g} U_{g}}
$$

and it would be 'exact' to estimate the 'impedance of interest' $Z_{\mathrm{r}}(\mathrm{j} \omega)$ from: 


$$
\widehat{Z}_{r}(j \omega)=\frac{S_{P U_{g}}(j \omega)}{S_{Q U_{g}}(j \omega)}
$$

However, it is supposed that the test is done in 'normal breathing conditions', which may result in an interference between the (unknown) breathing signal $U_{r}$ and the test signal $U_{g}$, making the identification exercise more difficult.

From the point of view of the forced oscillatory experiment, the signal components of respiratory origin, $\left(U_{r}\right)$ have to be regarded as pure noise for the identification task . Nevertheless, if the test signal $U_{g}$ is designed to be uncorrelated with the normal respiratory breathing signal $\mathrm{U}_{\mathrm{r}}$, then $S_{U_{r} U_{g}} \equiv 0$, and the approach (9) is still valid, based on (7) with $S_{U_{r} U_{g}} \equiv 0$. The measured data to be processed further in MatLab $^{\circledR}$ are: 1) the driving signal $U_{g}$ (pressure oscillations); 2) the trans-respiratory pressure $\mathrm{P}$; and 3) the air-flow Q. While the patient is breathing through the mouthpiece (biological filter - bf), the measurement is performed simultaneously by the data acquisition card and by the DSP board in the apparatus. The data is processed by the students using the two approaches in spectral analysis: correlation method and periodogram method, and a brief description of the algorithms is given in sections 5.1 and 5.2 of this paper.

The results are given by means of modulus-phase plot and real-imaginary parts plot respectively. From (Oostveen et al., 2003) it is known that the imaginary part depends on frequency, and accounts for the elastic and inertial properties of the mechanical model. The key concept of the forced oscillatory respiratory mechanics is the impedance $\left(Z_{\mathrm{r}}\right)$, the spectral (frequency domain) relationship between pressure (P) and airflow (Q). In simple terms, $Z_{\mathrm{r}}$ can be conceived as a generalisation of resistance, since it embodies both the in-phase and out-of-phase relationships between $\mathrm{P}$ and $\mathrm{Q}$. The inphase component is called the real part of $Z_{r}$ (or resistance $\left(\mathrm{R}_{\mathrm{r}}\right)$ ), whereas the out-of-phase relationship is expressed by the imaginary part (or reactance $\left(X_{r}\right)$ ), and both appear as functions of the frequency of oscillation (f). In other words, $R_{r}$ describes the dissipative mechanical properties of the respiratory system, whereas $X_{r}$ is related to the energy storage capacity.

\subsection{Spectral Analysis via Correlation Functions.}

The cross-correlation functions $\quad \hat{R}_{Q U_{g}}$ and $\hat{R}_{P U_{g}}$ between the driving signal and flow, and the driving signal and pressure respectively, can be computed as following:

$$
\begin{aligned}
& \hat{R}_{Q U_{g}}(\tau)=\frac{1}{N-\tau} \sum_{t=0}^{N-\tau-1} Q(t) U_{g}(t+\tau) \\
& \hat{R}_{P U_{g}}(\tau)=\frac{1}{N-\tau} \sum_{t=0}^{N-\tau-1} P(t) U_{g}(t+\tau)
\end{aligned}
$$

where: $\mathrm{N}=$ total number of measured samples; $\tau=$ $\mathrm{M}, \ldots,-1,0,1, \ldots \mathrm{M},(\mathrm{M}<<\mathrm{N}, \mathrm{M} \approx 0.1 \mathrm{~N})$.

Because the cross-correlation functions do not (necessarily) converge to zero, a discontinuity point will result in the estimates; therefore the use of window functions is compulsory in order to have an appropriate estimation. The 'windows' try to decrease the discontinuity at beginning and end of a data sequence; this is important because discontinuities introduce leakage frequencies. The most appropriate window function to use in our case is "Blackman", and it returns the N-point symmetric Blackman window in a column vector using the formula:

$$
w(n)=\left\{\begin{array}{l}
0.42-0.5 \cos \left(\frac{2 \pi n}{N-1}\right)+0.8 \cos \left(\frac{4 \pi n}{N-1}\right) \\
\text { for } 0 \leq n \leq N-1 \\
\text { and } 0 \text { otherwise }
\end{array}\right.
$$

Finally, the spectra vector is obtained by applying Fast Fourier Transform (FFT) to the vector of correlation function (11).

$$
\begin{aligned}
& S_{Q U_{g}}(k)=\sum_{i=0}^{M} \hat{R}_{Q U_{g}}(i) * e^{-j 2 \pi \frac{k i}{2 M+1}}+\sum_{i=-M}^{-1} \hat{R}_{Q U_{g}}(i) * e^{-j 2 \pi \frac{k i}{2 M+1}} \\
& S_{P U_{g}}(k)=\sum_{i=0}^{M} \hat{R}_{P U_{g}}(i) * e^{-j 2 \pi \frac{k i}{2 M+1}}+\sum_{i=-M}^{-1} \hat{R}_{P U_{g}}(i) * e^{-j 2 \pi \frac{k i}{2 M+1}}
\end{aligned}
$$

5.2 Spectral Analysis via Averaging Modified Periodograms.

Another approach to spectral analysis is to make use of the periodogram. The procedure consists in dividing the total number of samples $\mathrm{N}$ into $\mathrm{P}$ subintervals of length L; multiply each of them with an appropriate window $w(i)$ (Blackman), and apply FFT. Compute $\mathrm{P}$ modified periodograms:

$$
\begin{aligned}
& P_{P U_{g}}^{i}(k)=\frac{T_{s}}{L^{*} S c a l} U_{g}^{i}(k) * P^{i}(k) \\
& P_{Q U_{g}}^{i}(k)=\frac{T_{s}}{L^{*} \text { Scal }} U_{g}^{i}(k) * Q^{i}(k)
\end{aligned}
$$

where $i$ is the periodogram number $(i=1 \rightarrow \mathrm{P})$ and Scal $=\frac{1}{L} \sum_{k=0}^{L-1} w^{2}(k)$ is a scaling factor due to windowing. The spectral estimate is then obtained by averaging: 


$$
\begin{aligned}
& S_{P U_{g}}(k)=\frac{1}{P} \sum_{i=1}^{P} P_{P U_{g}}^{i}(k) \\
& S_{Q U_{g}}(k)=\frac{1}{P} \sum_{i=1}^{P} P_{Q U_{g}}^{i}(k)
\end{aligned}
$$

Because the FFT introduces periodicity, only the first half of the spectra vector is useful. The respiratory impedance is then following from relation (9). From the respiratory impedance, the modulus-phase dataset, or real-imaginary part data-set can be extracted. It is of most importance to plot the same frequency points in both experimental (=FOT device) and estimated data (=student's results). For this, a simple calculus is necessary in order to find the frequency resolution (accuracy). Knowing the sampling period $\mathrm{T}_{\mathrm{s}}$ and the length of the computed samples $\mathrm{M}(=256$, $512,1024,2048)$, the frequency resolution can be calculated as:

$$
f=\frac{1}{M^{*} T_{s}}
$$

In this way, the effect of increasing the number of computed samples in the spectral analysis procedures upon the resolution can be discussed. It is expected that the more computed points, the more accurate and thus noisier results will be obtained. A trade-off between accuracy and resolution will be needed.

The 'experimental' data of the respiratory impedance, is provided by the FOT device, in real $\left(\mathrm{R}_{\mathrm{r}}\right)$ and imaginary part $\left(\mathrm{X}_{\mathrm{r}}\right)$. From $\mathrm{R}_{\mathrm{r}}$ and $\mathrm{X}_{\mathrm{r}}$, the modulus and the phase can be obtained using the transformation formulas:

$$
\left|Z_{r}\right|=\sqrt{\left(R_{r}^{2}+X_{r}^{2}\right)} ; \Phi_{r}=\arctan \left(\frac{X_{r}}{R_{r}}\right)
$$

As a result, the students will become familiar with data manipulation in terms of interpretation possibilities and the necessary scaling from raw data - Volts (eg. Modulus must be in $\mathrm{dB}$, impedance is measured in $\mathrm{cmH}_{2} \mathrm{O} \mathrm{s} / \mathrm{L}$ ).

\section{EXPERIMENTAL RESULTS}

The results are compared as following: the experimental data from the FOT-device DSP board versus the estimated data via MatLab ${ }^{\circledR}$ programming procedures. In figures 5 and 6 are shown the results of the respiratory impedance by means of Bode plot and Impedance plot, respectively, for intervals of 256 computed samples. The respiratory impedance depicted in the following pictures is obtained via averaged modified periodograms and results are similar for the correlation method. The experimental data (from FOT device) is represented by circles and the estimated data via Matlab ${ }^{\circledR}$ by crosses.
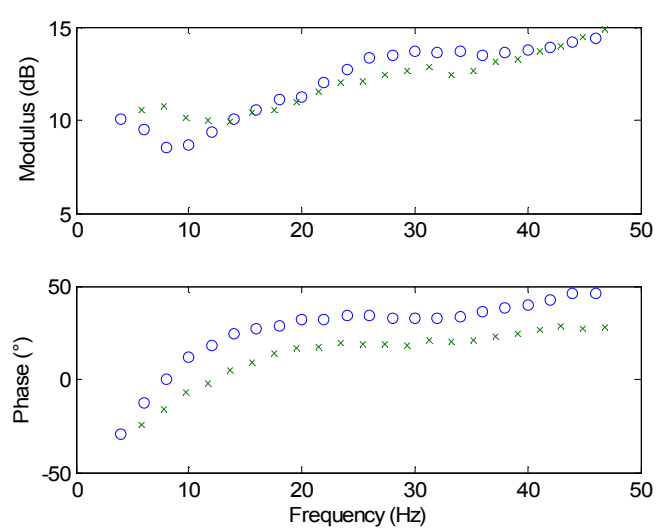

Fig. 5. Bode plot for intervals of 256 points.
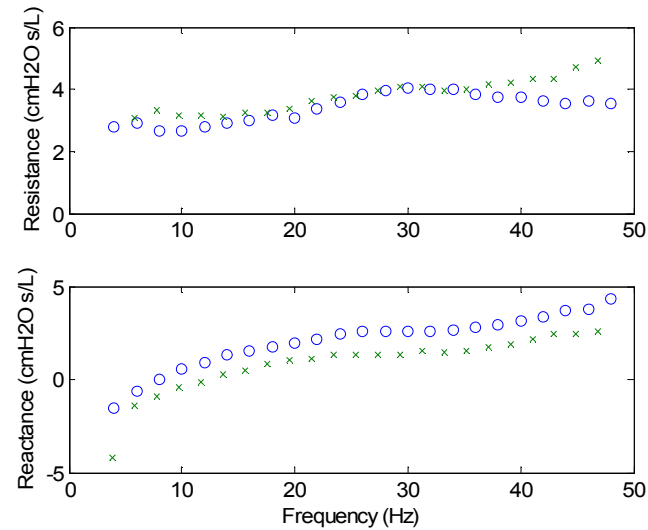

Fig. 6. Impedance plot for intervals of 256 points.
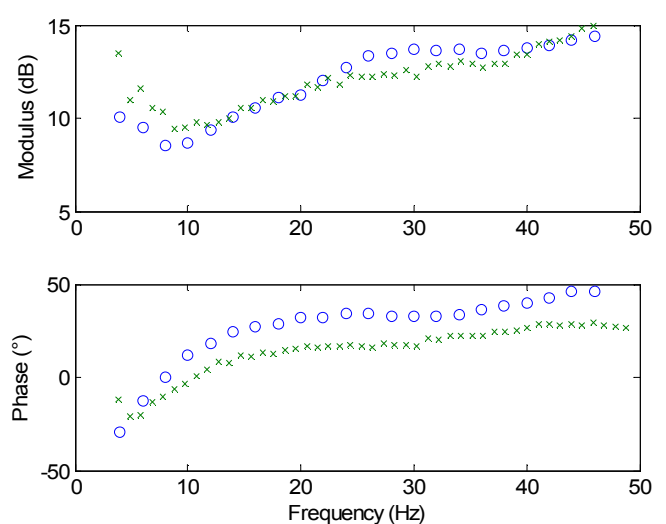

Fig. 7. Bode plot for intervals of 512 points.
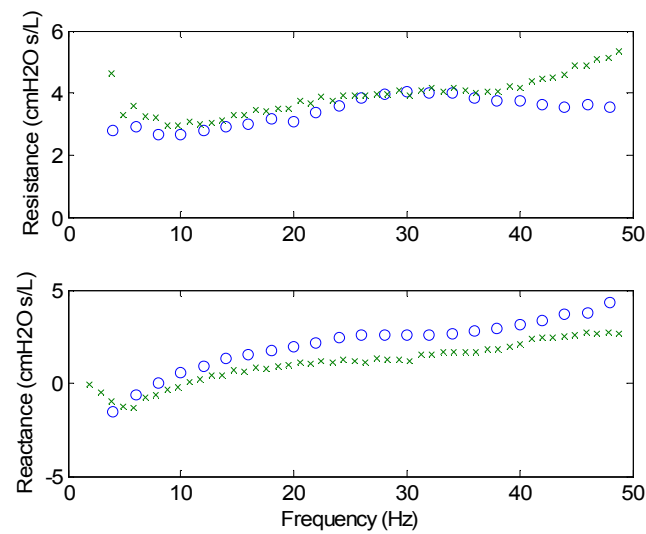

Fig. 8. Impedance plot for intervals of 512 points. 

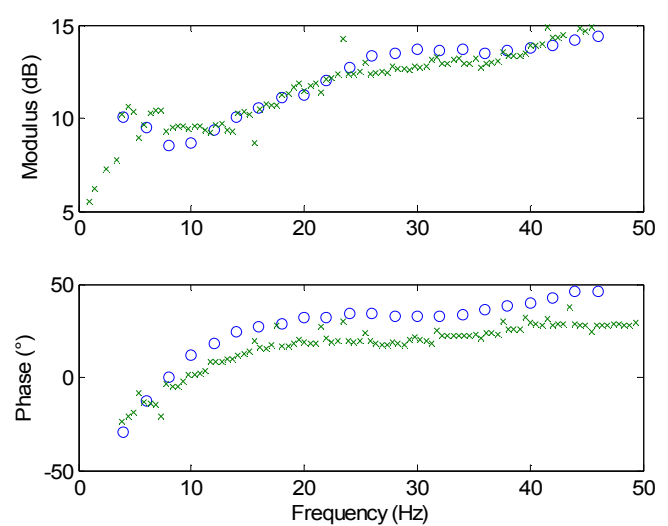

Fig. 9. Bode plot for intervals of 1024 points.
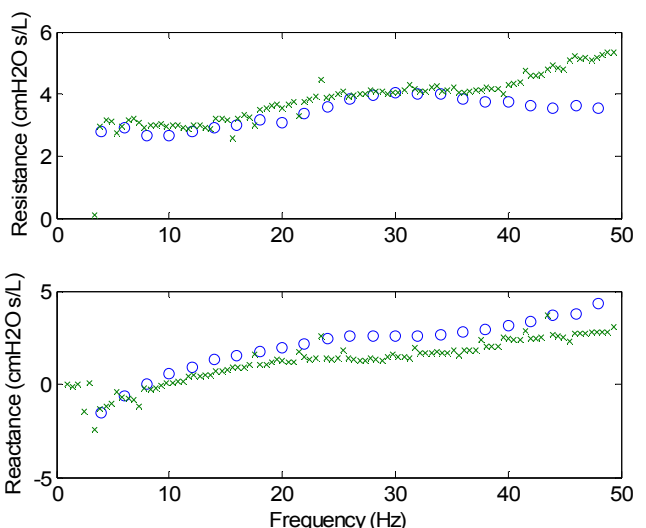

Fig. 10. Impedance plot for intervals of 1024 points.
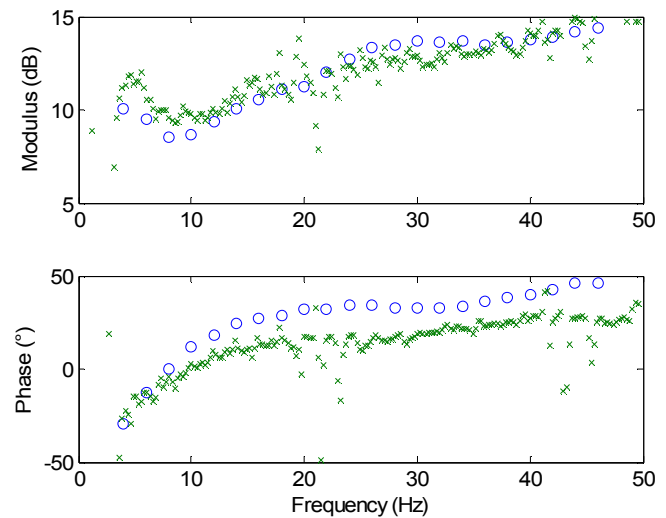

Fig. 11. Bode plot for intervals of 2048 points.
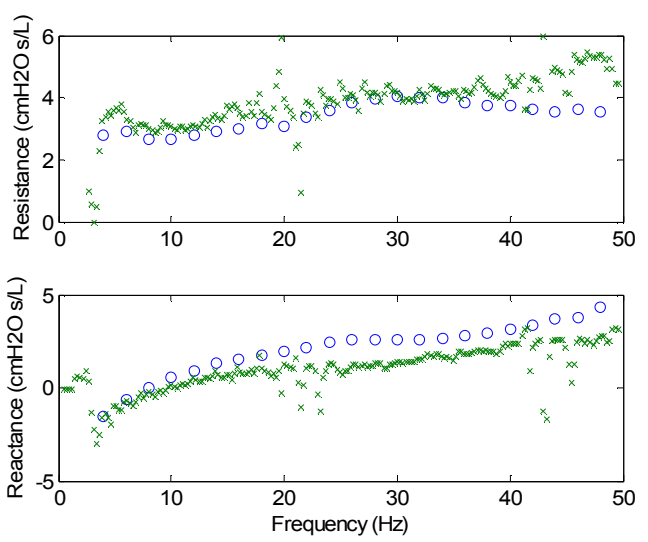

Fig. 12. Impedance plot for intervals of 2048 points.

Theoretically, increasing the number of computed samples in an interval increases also the noise amount in the estimations, as well as increasing the frequency resolution. For less number of computed samples in the intervals, the noise amount is decreasing, but also the resolution of estimates. If estimations are made for different interval lengths $(256,512,1024$ and 2048 - as in figures 5-12), it can be concluded that experimental results correspond to the theoretical insight. In this case, a trade-off between accuracy and resolution must be made. The length $\mathrm{L}=512$ of computed samples seems to be optimal in this application.

\section{CONCLUSIONS}

In this contribution, a project task and its model solution for non-parametric identification techniques is described. The aim of the project is to de-mystify the mathematical description of spectral analysis and to reduce its abstractness. To make the application more attractive to the students, a non-invasive lung function test is used to gather data upon their own respiratory mechanics. Although a diagnostic is not intended, some mechanical properties of the respiratory system can be discussed and compared to a standard for healthy patients (Oostveen et al., 2003; Navajas and Farré, 1999).

The students are trained to deal with real-life problems such as determining an optimal length for the data measurements in order to perform good quality estimation via averaged periodogram method. Data pre-processing is also an important aspect in system identification and is a crucial step for obtaining good estimations (e.g. ensure zero mean value for each data variable). Students are gathering experience to decide upon trade off between resolution and accuracy.

Finally, offering such laboratory experiments to the students leads to an increase in their motivation, interest and training in real-life applications.

\section{REFERENCES}

Ljung, L. (1999). System Identification, NJ: Prentice Hall.

Navajas, D., Farré, R., (1999). Oscillations mechanics, Eur Resp Mon, 12, 112-140

Oostveen, E., Macleod, D., Lorino, H., Farré, R., Hantos, Z., Desager, K., Marchal, F., (2003). The forced oscillation technique in clinical practice: methodology, recommendations and future developments, Eur Respir J, 22, 1026-1041

Van Noord, J.A., (1990). Oscillation mechanics of the respiratory system: Clinical applications and modelling, Doctoral Thesis, Katholieke Universiteit Leuven, 189p

Wellstead, P.E. (1990). Teaching Control with Laboratory Scale Models. IEEE Trans on Education, 33, 285-290 